\title{
Can PCSK7 be A New Pharmaceutical Target?
}

\author{
Masa-aki Kawashiri
}

Department of Cardiovascular Medicine, Graduate School of Medical Sciences, Kanazawa University, Kanazawa, Japan

\section{See article vol. 29: 000-000}

The natural history of nonalcoholic fatty liver disease (NAFLD) is characterized by progression from simple steatosis (fatty change), which affects more than $5 \%$ of hepatocytes, to nonalcoholic steatohepatitis (NASH), and subsequently fibrosis, cirrhosis, and hepatocellular carcinoma. An increasing number of patients suffered from NAFLD-associated cancer without cirrhosis. A recent meta-analysis showed that the prevalence of NAFLD is estimated to be more than $25 \%$ in the Japanese population aged over 50 years and in the American population aged over 20 years $^{1)}$. Patients with NAFLD frequently suffer from overweightedness, dyslipidemia, and type 2 diabetes mellitus, which may result in atherosclerotic cardiovascular diseases.

NAFLD is based on the disruption of energy homeostasis due to overnutrition. However, more than $20 \%$ of NAFLD can occur in normal people or those with lean body weight, especially in the Japanese population. Moreover, the severity of NASH is not always correlated with that of steatosis as evaluated via liver biopsy or imaging, which is the reflection of the amount of triglyceride in the hepatocytes. Some genetic and epigenetic factors as well as environmental factors are known to be associated with the development of NAFLD and NASH. Genome-wide association studies revealed that polymorphisms in patatin-like phospholipase domain-containing 3 (PNPLA3), transmembrane 6 superfamily member 2 (TM6SF2), and member bound O-acyltransferase domain-containing 7 (MBOAT7) contribute to the development of NAFLD and other liver diseases ${ }^{2)}$. PNPLA3 is a triacylglycerol lipase that mediates triacylglycerol hydrolysis in adipocytes and is strongly associated with hepatic steatosis and fibrosis. TM6SF2 and MBOAT7 are also known to regulate the secretion of very-low-density lipoprotein from hepatocytes and the accumulation of lipid in hepatocytes. An abnormal metabolism of iron (dysmetabolic hyperferritinemia) represents another typical feature of NAFLD ${ }^{3)}$. Although the underlying mechanism remains unknown, iron deposition in hepatocytes is associated with a more severe liver damage in patients with NAFLD.

The proprotein convertase (PC) family plays important roles in human homeostasis and pathophysiological states, including endocrinopathies, atherosclerosis, infectious diseases, cancer, and neurodegenerative diseases ${ }^{4)}$. Thus, PCs may have the potential to be therapeutic targets of several pathophysiological conditions. They are synthesized as inactive precursors and modified by several proteases to develop the original activities. The prodomains of PCs contribute to the folding and activation of the protein and participate in the intramolecular autocatalytic cleavage; thereafter, the cores of PCs emerge from the endoplasmic reticulum. Finally, the protein is activated through a $\mathrm{pH}$ - and calciumdependent cleavage event in the trans-Golgi networkendosomal compartments ${ }^{4}$. PC subtilisin/kexin type 9 (PCSK9) is the ninth member of the PC family and was found to be the third causal gene of autosomal dominant hypercholesterolemia ${ }^{5)}$. PCSK9 has been one of the most successful therapeutic targets in terms of efficacious drug development (PCSK9 inhibitors) less than 10 years after its discovery. PCSK9 inhibitors are able to cause a significant reduction in low-density lipoprotein cholesterol and cardiovascular events. In this issue of the Journal of Atherosclerosis and Thrombosis, Furuhashi et al. investigated the association between the plasma levels of PC subtilisin/ kexin type 7 (PCSK7) and several metabolic parameters in the general Japanese population without any medications ${ }^{6}$. PCSK7, another member of the PC family, is a ubiquitously expressed transmembrane

Address for correspondence: Masa-aki Kawashiri, Department of Cardiovascular Medicine, Graduate School of Medical Sciences, Kanazawa University, 13-1 Takara-machi, Kanazawa, 920-8641, Japan E-mail: mk@med.kanazawa-u.ac.jp

Received: December 3, 2021 Accepted for publication: December 15, 2021

Copyright@2022 Japan Atherosclerosis Society

This article is distributed under the terms of the latest version of CC BY-NC-SA defined by the Creative Commons Attribution License. 
protease and shares the substrates with the other PCs, especially Furin ${ }^{4}$. Of those, the transferrin receptor 1 was identified as a specific substrate of PCSK7. Both PCSK7 and Furin are localized in the trans-Golgi network, and only a small fraction is found at the cell surface $^{4)}$. On the contrary, the prosegment of PCSK7, which is an inhibitor of full PCSK7, is secreted from the cell and is known to mask the catalytic site of PCSK7 itself ${ }^{4)}$. Furuhashi et al. found that the plasma level of PCSK7 was significantly and independently associated with the body mass index, logarithmically transformed triglycerides, and logarithmically transformed fatty liver index, which is calculated using body height, body weight, waist circumference, and levels of gamma-glutamyl transpeptidase and triglycerides ${ }^{6}$.

As this is a cross-sectional study, it is difficult to show the causality between PCSK7 and NAFLD or dyslipidemia. Moreover, this study evaluated hepatic steatosis not by histology or imaging but by noninvasive numerical index. The epitope of the ELISA kit for PCSK7 measurement used in this study was not described, and the correlation between the plasma levels and biological activities of PCSK7 was unknown. Despite the above limitations, the first data on PCSK7 levels and metabolic parameters obtained from general healthy Japanese population are valuable. Moreover, previous studies demonstrated the potential function of PCSK7 in inactivating lipoprotein lipase through the degradation of apolipoprotein $\mathrm{A}-\mathrm{V}$, an indirect activator of lipoprotein lipase ${ }^{7)}$, and cleaving and activation of angiopoietin-like protein 4, which is a lipoprotein lipase inhibitor ${ }^{8)}$. Transferrin receptor 1 , which is a carrier protein of iron into the cell, is a specific substrate of PCSK7, and iron overload is known to be associated with the development and worsening of NAFLD.

It is an interesting question whether PCSK7 could be a new pharmaceutical target for NAFLD and dyslipidemia. To answer this question, genetic interventional animal study and Mendelian randomization study would be helpful. PCSK7 knockout mice are at least viable, although their plasticity of responding was reported to be mildly abnormal $^{9}$. Recent genome-wide association studies revealed that the missense variant in the PCSK 7 gene was associated with plasma triglyceride levels ${ }^{10}$. Moreover, it was elucidated that the PCSK7 rs 236918 gain-of-function variant was associated with dyslipidemia and more severe liver disease in high-risk patient at NAFLD ${ }^{11)}$. Thus, PCSK7 inhibition may be a promising strategy to prevent NAFLD and dyslipidemia and eventually prevent atherosclerotic cardiovascular diseases.
For instance, the inhibitor of cholesterol ester transfer protein (CETP) encountered many hurdles in becoming a pharmaceutical candidate. A significant reduction in cardiovascular events in subjects with rare variants of the CETP gene (CETP deficiency) promotes the trend of CETP inhibitor reproduction again $^{12,13)}$. So, far, there has been no report about human PCSK7 deficiency. If it is proven that individuals with PCSK7 deficiency are healthy and less frequently suffer from NAFLD or dyslipidemia, then PCSK7 inhibitor will be strongly pushed forward to be a candidate of pharmaceutical target in the future.

\section{Conflict of Interest}

None.

\section{References}

1) Diel AM, Day C. Cause, Pathogenesis, and treatment of nonalcoholic steatohepatitis. N Engl J Med, 2017; 377: 2063-2072

2) Anstee QM, Seth D, Day CP. Genetic Factors that affect risk of alcoholic and nonalcoholic fatty liver disease. Gastroenterology, 2016; 150: 1728-1744.e7

3) Valenti L, Fracanzani AL, Bugianesi E, Dongiovanni P, Galmozzi E, Vanni E, Canavesi E, Lattuada E, Roviaro G, Marchesini G, Fargion S. HFE genotype, parenchymal iron accumulation, and liver fibrosis in patients with nonalcoholic fatty liver disease. Gastroenterology, 2010; 138: 905-912

4) Artenstein AW, Opal SM. Proprotein convertases in health and disease. N Engl J Med, 2011; 365: 2507-2518

5) Abifadel M, Varret M, Rabès JP, Allard D, Ouguerram K, Devillers M, Cruaud C, Benjannet S, Wickham L, Erlich D, Derré A, Villéger L, Farnier M, Beucler I, Bruckert E, Chambaz J, Chanu B, Lecerf JM, Luc G, Moulin P, Weissenbach J, Prat A, Krempf M, Junien C, Seidah NG, Boileau C. Mutations in PCSK9 cause autosomal dominant hypercholesterolemia. Nat Genet, 2003; 34: 154-156

6) Furuhashi M, Kataoka Y, Nishikawa R, Koyama M, Sakai A, Higashiura Y, Tanaka M, Saitoh S, Shimamoto K, Ohnishi H. Circulating PCSK7 Level is Independently Associated with Obesity, Triglycerides Level and Fatty Liver Index in a General Population without Medication. J Atheroscler Thromb, 2021 (Online ahead of print) doi: http://doi.org/10.5551/jat.63159

7) Ashraf Y, Duval S, Sachan V, Essalmani R, Susan-Resiga D, Roubtsova A, Hamelin J, Gerhardy S, Kirchhofer D, Tagliabracci VS, Prat A, Kiss RS, Seidah NG. Proprotein convertase 7 (PCSK7) reduces apoA-V levels. FEBS J, 2020; 287: 3565-3578

8) Lei X, Shi F, Basu D, Huq A, Routhier S, Day R, Jin W. Proteolytic processing of angiopoietin-like protein 4 by proprotein convertases modulates its inhibitory effects on lipoprotein lipase activity. J Biol Chem, 2011; 286:

\section{Advance Publication Journal of Atherosclerosis and Thrombosis}


$15747-15756$

9) Wetsel WC, Rodriguiz RM, Guillemot J, Rousselet E, Essalmani R, Kim IH, Bryant JC, Marcinkiewicz J, Desjardins R, Day R, Constam DB, Prat A, Seidah NG. Disruption of the expression of the proprotein convertase PC7 reduces BDNF production and affects learning and memory in mice. Proc Natl Acad Sci U S A, 2013; 110 : $17362-17367$

10) Kurano M, Tsukamoto K, Kamitsuji S, Kamatani N, Hara M, Ishikawa T, Kim BJ, Moon S, Jin Kim Y, Teramoto T. Genome-wide association study of serum lipids confirms previously reported associations as well as new associations of common SNPs within PCSK7 gene with triglyceride. J Hum Genet, 2016; 61: 427-433

11) Dongiovanni P, Meroni M, Baselli G, Mancina RM, Ruscica M, Longo M, Rametta R, Cespiati A, Pelusi S, Ferri N, Ranzani V, Nobili V, Pihlajamaki J, Fracanzani AL, Badiali S, Petta S, Fargion S, Romeo S, Kozlitina J, Valenti L. PCSK7 gene variation bridges atherogenic dyslipidemia with hepatic inflammation in NAFLD patients. J Lipid Res, 2019; 60: 1144-1153
12) Nomura A, Won HH, Khera AV, Takeuchi F, Ito K, McCarthy S, Emdin CA, Klarin D, Natarajan P, Zekavat SM, Gupta N, Peloso GM, Borecki IB, Teslovich TM, Asselta R, Duga S, Merlini PA, Correa A, Kessler T, Wilson JG, Bown MJ, Hall AS, Braund PS, Carey DJ, Murray MF, Kirchner HL, Leader JB, Lavage DR, Manus JN, Hartze DN, Samani NJ, Schunkert H, Marrugat J, Elosua R, McPherson R, Farrall M, Watkins H, Juang JJ, Hsiung CA, Lin SY, Wang JS, Tada H, Kawashiri MA, Inazu A, Yamagishi M, Katsuya T, Nakashima E, Nakatochi M, Yamamoto K, Yokota M, Momozawa Y, Rotter JI, Lander ES, Rader DJ, Danesh J, Ardissino D, Gabriel S, Willer CJ, Abecasis GR, Saleheen D, Kubo M, Kato N, Ida Chen YD, Dewey FE, Kathiresan S. ProteinTruncating Variants at the Cholesteryl Ester Transfer Protein Gene and Risk for Coronary Heart Disease. Circ Res, 2017; 121: 81-88

13) Nurmohamed NS, Ditmarsch M, Kastelein JJP. CETPinhibitors: from HDL-C to LDL-C lowering agents? Cardiovasc Res, 2021 (Online ahead of print) 